\title{
INFLUENCIA DEL ENSILADO BIOLOGICO DE PESCADO Y PESCADO COCIDO EN EL CRECIMIENTO Y LA COMPOSICION CORPORAL DE ALEVINOS DE GAM ITANA Colossoma macropomum ${ }^{1}$
}

\author{
Palmira Padilla Pérez* \\ Manoel Pereira-Filho** \\ L uis M ori Pinedo**
}

\section{RESUMEN}

Se compararon los efectos de dos fuentes proteicas, ensilado biológico de pescado y pescado cocido, producidas con residuos del fileteado de manitoa Brachyplatystoma vaillantii, a través del crecimiento y de la composición corporal de alevinos de gamitana Colossoma macropomum en 85 días experimentales. Se elaboraron cuatro raciones con tenores de proteína de 24,7 a $27,0 \%$ y energía bruta entre 438,9 y $445,4 \mathrm{Kcal} / 100 \mathrm{~g}$ de materia seca. El experimento fue conducido en 20 tanques de cemento-amianto con capacidad para 250 litros, cada uno con 12 alevinos, con longitud total de $10,33 \mathrm{~cm}$ y peso medio de $27,42 \mathrm{~g}$. Los peces fueron aclimatados a condiciones experimentales durante 10 días.

La alimentación se realizó dos veces al día a razón de $3 \%$ de la biomasa, reajustándose la cantidad de alimento cada 28 días, luego del muestreo de crecimiento en peso.

La composición corporal de los peces se determinó al inicio y al final del experimento, determinándose mediante análisis estadísticos que los tratamientos no presentaron influencia significativa $(P>0,05)$ en el peso final y en la composición corporal de los alevinos de gamitana.

Palabras clave: Gamitana, ensilado de pescado, proteína, fuentes proteicas.

1 Parte de la Disertación de M aestría. Instituto de Pesquisas da A mazonia - INPA. Manaus/AM Brasil.

* Investigadora del Instituto de Investigaciones de la A mazonía Peruana.

** Investigador del Instituto $\mathrm{N}$ acional de pesquisas da A mazonia.

*** Investigador de la U niversidad N acional de la A mazonía Peruana (UNAP). 


\section{ABSTRACT}

Two fish protein sources (biological fish silage and cooked by-product of fish, remains of manitoa Brachyplatystoma vaillantii were evaluated on growth performance and body composition of alevins gamitana Colossoma macropomumin 85 days experiment.

The protein and energy levels varied from 24,7 to $27,0 \%$ and from 438,9 to $445,4 \mathrm{~K} \mathrm{cal} / 100$ dry matter respectively. The experiment was conduced in 20 asbestos tanks, with $250 \mathrm{I}$ capacity stocked with 12 fish (mean initial weigth and length $27,42 \mathrm{~g}$ and $10,33 \mathrm{~cm}$ respectively).

The fish were adapted to experimental conditions for 10 days. Fish were fed twice daily ( $3 \%$ body weight). Proximate analyses were varied to the feed and to the fish at the begining at the end of the experiment. Five replicate tanks were use for each dietary treatament. The ANOVA showed that the sources had no significant diferences $(P>0,05)$ on final weigth and body composition of alevins gamitana.

Key-words: Gamitana, fish silage, protein, protein source.

\section{INTRODUCION}

En la región amazónica la producción pesquera es alta y solo una pequeña fracción del stock es explotada (Bayley \& Petrere Jr., 1989). En 1987, la producción de pescado en el estado del Amazonas fue alrededor de 45,5 mil toneladas, mientras que en el año siguiente disminuyo a 38,7 mil toneladas (SU DEPE/AM , 1988). J unk \& Honda (1976) afirman que $20 \%$ de las capturas se pierden por la falta de almacenamiento. A simismo, el pescado que pasa por un proceso de industrialización, pierde por lo menos $40 \%$ de su peso en la forma de residuos.

Una de las alternativas viables es el aprovechamiento de estas pérdidas en la elaboración de ensilados (proteína hidrolizada) y otros productos, cuya mayor importancia está en su utilización para la formulación de raciones de bajo costo y al to valor nutricional. 
Varios autores vienen resaltando la ventaja de la utilización de la proteína hidrolizada de pescado sobre la harina de pescado en el crecimiento de animales (M cBride et al., 1968; Wignall \& Tatterson, 1977; V an Wyk et al., 1977; Villela De A ndrade, 1982; A rthur, 1991; X imenes-Carneiro, 1991 y Lessi et al., 1992). Dentro de las muchas especies de peces amazónicos que pueden ser cultivadas, destaca la gamitana, Colossoma macropomum, especie con gran potencial para la piscicultura (M acedo, 1979; Lovshin, 1980; Goulding \& Carvalho, 1982; Da Silva et al., 1984; Saint-Paul, 1984; Saldaña \& López, 1988).

Este trabajo tuvo como objetivo comparar los efectos de dos fuentes proteicas (ensilado biológico de pescado y pescado cocido) a través del desarrollo en peso y longitud y la composición corporal de alevinos de gamitana, Colossoma macropomum.

\section{MATERIAL Y METODOS}

El ensilado biológico de pescado y el pescado cocido utilizados en este estudio fueron preparados a partir de residuos del fileteado de manitoa, Brachyplatystoma vaillantii. Para la preparación del ensilado, se utilizaron las recomendaciones de FA O (1985).

Fueron formuladas cuatro raciones, en las cuales la harina de soya, harina de maíz, harina de trigo, las vitaminas y los minerales se mantuvieron constantes, mientras el ensilado biológico de pescado y el pescado cocido variaron en proporción inversa de 0 a $36 \%$ del total de las raciones (Tabla 1).

Los peces fueron alimentados diariamente a razón de $3 \%$ de la biomasa de cada tanque.

En este experimento fueron usados 20 tanques cilíndricos de cemento-amianto con capacidad de 250 litros cada uno. La tasa de carga fue de 12 peces por tanque. El período de adaptación de los peces a las condiciones experimentales fue de diez días, al cabo de los cuales se realizó un primer muestreo, que se repitió tres veces a intervalos de 28 días.

Se efectuaron análisis bromatológicos de los peces y de las raciones antes de iniciar el experimento, y de los peces al término del experimento, según las recomendaciones de la A.O.A.C., 1975. 
El monitoreo de los factores físico-químicos del agua de los tanques se realizó a través de mediciones diarias de la temperatura y determinaciones semanales de los tenores de oxígeno disuel to y de la conductividad eléctrica.

Se utilizó el diseño experimental totalmente randomizado, con cuatro tratamientos y cinco repeticiones de acuerdo con Banzatto \& Kronka (1989), para determinar los efectos de las raciones sobre el crecimiento en longitud y en peso, a la vez que en la composición corporal de los peces. Se consideró como unidad experimental, la media de los datos de los peces de cada tanque.

TABLA 1. Composición porcentual de raciones experimentales

\section{R A C I O O N E S}

\begin{tabular}{lrrrr}
\hline Ingredientes & $\mathbf{R} \mathbf{R}$ & $\mathbf{R 2}$ & $\mathbf{R 3}$ & $\mathbf{R 4}$ \\
\hline Ensilado biológico & 36,00 & 24,00 & 12,00 & 00,00 \\
Pescado cocido & 00,00 & 12,00 & 24,00 & 36,00 \\
H arina de soya & 30,00 & 30,00 & 30,00 & 30,00 \\
H arina de maíz & 28,00 & 28,00 & 28,00 & 28,00 \\
H arina de trigo & 5,00 & 5,00 & 5,00 & 5,00 \\
Vitaminas & 0,50 & 0,50 & 0,50 & 0,50 \\
M inerales & 0,50 & 0,50 & 0,50 & 0,50 \\
\hline
\end{tabular}

\section{RESULTADOS}

En la Tabla 2 se verifica que el ensilado posee valores más bajos para proteínas y lípidos, en relación al pescado cocido, y éste presenta mayor valor calórico. 
TABLA 2. Composición bromatológica de las raciones experimentales, del ensilado biológico y del pescado cocido

Nutrientes

(g/ $100 g$ de MS)

\section{Raciones}

\begin{tabular}{llll}
\hline R1 & R2 & R3 & R4
\end{tabular}
Ensilado

Pescado

Cocido
M ateria seca

Proteína Bruta

Extracto etéreo

Ext. no nitrogen.

M aterial mineral

Fibra bruta

Energía bruta

(kcal EB/100g M S)
$24,7 \quad 25,9$

6,4

6,4

59,2

56,0

$7,9 \quad 10,1$

1,8

1,6

445,4

En la tabla 3 se observa que los peces en los tratamientos, presentaron un menor valor de proteína bruta y mayor valor de extracto etéreo, que al inicio del experimento, en tanto que, los valores de extracto no nitrogenado en los tratamientos 1, 2 y 3, se mantuvieron en sus niveles, con una ligera disminución en el tratamiento cuatro, sin tener variación significativa entre ellos $(P>0.05)$.

TABLA 3. Análisis bromatológico de peces de los diferentes tratamientos al inicio y al final del experimento.

\begin{tabular}{|c|c|c|c|c|c|c|}
\hline \multirow[b]{2}{*}{ Nutrientes } & \multirow{2}{*}{\multicolumn{2}{|c|}{$\begin{array}{l}\text { Composición } \\
\text { Inicial }\end{array}$}} & \multicolumn{3}{|c|}{$\begin{array}{l}\text { Composición final } \\
\text { (Tratamientos) }\end{array}$} & \multirow[b]{2}{*}{$\mathbf{T} 4$} \\
\hline & & & $\mathrm{T} 1$ & T2 & T3 & \\
\hline Humedad & 70,00 & 66,80 & \multicolumn{3}{|c|}{ ( $\mathrm{g} / 100 \mathrm{~g}$ de materia seca) } & \\
\hline $\begin{array}{l}\text { Proteína bruta } \\
\text { Extracto etéreo } \\
\text { M aterial mineral } \\
\text { Extracto no nitrogenado }\end{array}$ & & $\begin{array}{r}54,30 \\
22,40 \\
16,20 \\
7,10\end{array}$ & $\begin{array}{r}43,46 \\
38,30 \\
10,40 \\
7,84\end{array}$ & $\begin{array}{r}42,70 \\
38,42 \\
11,12 \\
7,76\end{array}$ & $\begin{array}{r}42,34 \\
40,06 \\
10,12 \\
7,48\end{array}$ & $\begin{array}{r}43,92 \\
36,68 \\
12,64 \\
6,76\end{array}$ \\
\hline
\end{tabular}


La temperatura del agua, durante el estudio, presentó un valor máximo de $28,9^{\circ} \mathrm{C}$ y un valor mínimo de $24,7^{\circ} \mathrm{C}$. La mayor diferencia encontrada entre los dos períodos fue de $4,2^{\circ} \mathrm{C}$. L a amplitud de variación de este parámetro por la mañana fue de $3,2^{\circ} \mathrm{C}$ y de la tarde fue de $1,9^{\circ} \mathrm{C}$. (F igura 1 ). Los valores del $\mathrm{pH}$ variaron entre 5,5 y 6,7 , definiendo un carácter "ácido". De un modo general, los niveles de oxígeno disuel to variaron de 6,6 a 6,8 mg/l. Estos valores se mantuvieron casi constantes por causa de la renovación del agua. Los valores de la conductividad eléctrica del agua variaron entre 118,3 a 120,5 S/cm.

Los datos del desempeño de los peces alimentados con las diferentes raciones aparecen en la Figura 2. Se puede constatar que la mayor ganancia de peso ocurrió en el tratamiento (T1) en que los peces fueron alimentados con la ración $R 1$. La ganancia de peso diaria de los peces presentó la tendencia siguiente: T1> T4> T2$>$ T3. Las medias de la ganancia en peso analizadas a través del análisis de varianza, revelaron mediante el test $F$, un efecto estadísticamente no significativo $(P>0,05)$ entre los tratamientos. 
Figura № 1. Promedio diarios de temperatura del agua en los estanques experimentales

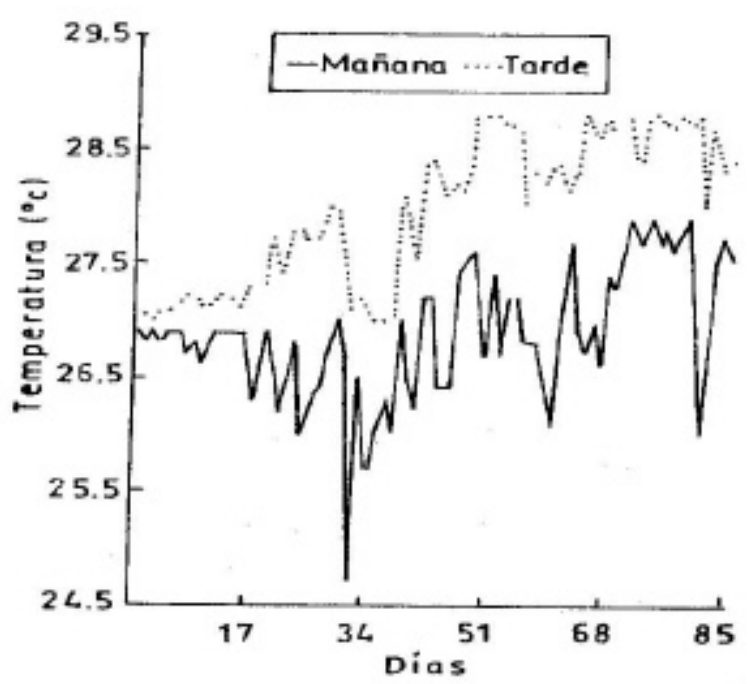

Figura № 2. Crecimiento en peso de los peces

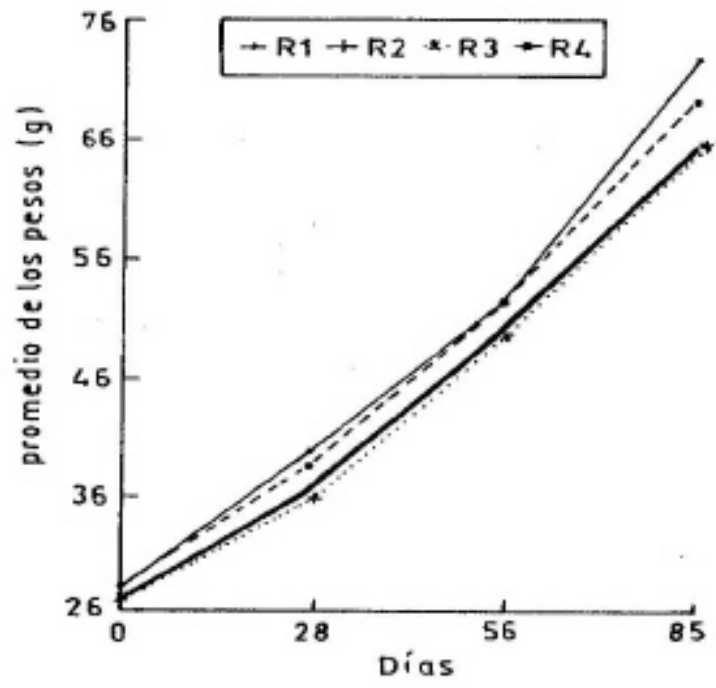


Los valores de la tasa media de conversión alimentaria aparente de los peces, demostraron que el tratamiento T1 (R1) fue mejor en comparación a los otros tratamientos, donde se muestran valores desde 2,1:1 a 2,3:1, que se consideran satisfactorios.

Durante todo el experimento la tasa de sobrevivencia de los peces fue $100 \%$, a pesar del manipuleo mensual y de los acentuados cambios de los parámetros físicosquímicos del agua de los tanques.

TABLA 4. Ganancia media de peso (g), error standard de la media (g) de los peces sometidos a los diferentes tratamientos

$\begin{array}{cccccc} & 1 \underline{a} & 2 \underline{a} & 3 \underline{a} & 4 \underline{a} & \text { G anancia } \\ \text { Trat. } & \text { pesaje } & \text { pesaje } & \text { pesaje } & \text { pesaje } & \text { Peso g/día }\end{array}$

T1 $27,78 \pm 0,75$

$39,75 \pm 2,24$

$55,27 \pm 3,41$

$72,48 \pm 6,41$

0,52

$\mathrm{T} 2$

$26,80 \pm 0,36$

$36,67 \pm 2,14$

$50,24 \pm 4,72$

$65,38 \pm 7,40$

0,45

T3

$27,02 \pm 0,71$

$35,92 \pm 1,13$

$49,45 \pm 2,16$

$64,92 \pm 3,70$

0,44

T4

$28,08 \pm 1,17$

$38,71 \pm 2,42$

$52,28 \pm 3,87$

$69,43 \pm 6,28 \quad 0,48$

\section{DISCUSION}

Los valores de la composición bromatológica del ensilado biológico (Tabla 2), tienen similitud con los valores encontrados por Ximenes-Carneiro (1991), diferenciándose apenas en los tenores de grasa y de fibra bruta. El hecho de la utilización de diferentes materias primas y las diferentes técnicas usadas en la elaboración de los ensilados, muestra mucha divergencia en su composición y a la vez puede variar por la especie, por la época de captura, por el hábito alimentario, estadío del pez utilizado, etc. Los valores calóricos reportados por diversos autores,

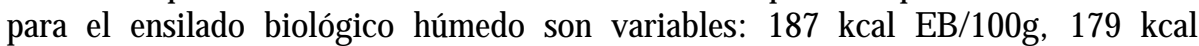
$E B / 100 \mathrm{~g}$ y $114 \mathrm{kcal} E B / 100 \mathrm{~g}$, A reche et al. (1989), Ximenes-Carneiro (1991) y Lessi et al. (1992), respectivamente. Para el ensilado semi-seco X imenes-Carneiro

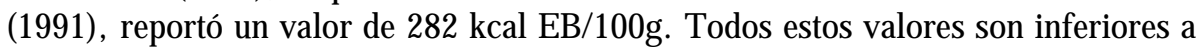
los valores encontrados en este trabajo. 
De igual forma los valores calóricos del pescado cocido fueron mayores que los encontrados en ensilado. Comparando los resultados de los análisis bromatológicos del pescado cocido y del ensilado semi-seco, se observa que los tenores de proteína, lípidos y el valor calórico del pescado cocido fueron mayores a los encontrados en el ensilado biológico semi-seco utilizados en el presente experimento.

Las variaciones de la temperatura y de la conductividad eléctrica del agua de los tanques experimentales tuvieron valores similares a los encontrados por XimenesCarneiro (1991) que trabajó en condiciones semejantes.

Los valores mínimos de oxígeno disuelto, dieron las condiciones ambientales adecuadas para el cultivo de la especie en estudio, de acuerdo a Val (1986) y Castagnolli (1992). Según Braum \& Junk (1982) y Saint-Paul (1984), la gamitana puede tolerar bajas concentraciones de oxígeno disuelto pudiendo sobrevivir en aguas con tenores de $0,5 \mathrm{mg} / \mathrm{l}$, empleando estrategias propias de la especie.

En la Tabla 4 se presentan los resultados de la ganancia de peso medio (g) y el error standard de las medias de los peces sometidos a los diferentes tratamientos. Al inicio y al final del experimento, el análisis de varianza de los pesos no presentó diferencia significativa $(P>0,05)$. En la conversión alimentaria aparente de los peces, la ración $\mathrm{R} 1$ mostró tener la mejor tasa $(2,1: 1)$, siendo este resultado similar con los obtenidos por Werder \& Saint-Paul (1979), Eckmann (1987) y XimenesCarneiro (1991). Los análisis de la composición bromatológica de los peces realizadas al inicio y al final del experimento (Tabla 3) mostraron que ocurrieron variaciones de lípidos y carbohidratos. Asimismo, las proteínas mantuvieron tenores similares en todos los tratamientos. Los niveles de carbohidratos en la composición final se debieron a la cantidad de harina usada, tanto en la elaboración del ensilado, como en la elaboración de las raciones. Parte de esos carbohidratos fueron transformados en lípidos de reserva, concordando con los resultados obtenidos por Cantelmo \& Sousa (1987), Eckmann (1987), Ximenes-Carneiro (1991) y M ori-Pinedo (1993). En nuestro experimento, los peces alimentados con las raciones a base del ensilado biológico de pescado y de pescado cocido, después de los análisis estadísticos, mostraron que no hubo diferencia significativa ( $P$ > $0,05)$. 


\section{BIBLIOGRAFIA}

ARECHE, N.T.; BERENZ, Z.V. y LEON, G.O. 1989. Desarrollo de ensilado de residuo de pescado utilizando bacterias lácticas del yogur. En: Consulta de Expertos sobre Tecnología de Productos Pesqueros en América Latina. 2. Montevideo. Roma, FA 0. 14p.

ARTHUR, L.M.S.R. 1991. Utilização de ensilado biológico de pescado na elaboração de uma ração para desenvolvimento de pós-larvas de camarão de água doce, Macrobrachium rosemberguii, M. Dissertação de M estrado. Rio de Janeiro: UFRRJ. 138pp.

ASSOCIATION OF OFFICIAL ANALYTICAL CHEMISTS - A.O.A.C. 1975. Official Methods of A nalysis. 12a. Edition. George Banta Co. INC., M anasha, Wisconsin, $937 \mathrm{pp}$.

BANZATTO, D.A. \& KRONKA, S. DO N. 1989. Experimentação Agrícola. Departamento de Ciências Exatas. Faculdade de Ciências Agrárias e Veterinárias - UNESP. Jaboticabal. SP. UNESP. 247 pp.

BAYLEY, P.B. \& PETRERE Jr, M. 1989. A mazon Fisheries: assessment methods, current status and managment options. In: Can. Spec. Publ. Fish. Aquat. Sci. 106: 385-398.

BRA U M, E. \& JUNK, W.J. 1982. Morphological A daptation of two A mazonian Characoides (Pisces) For Survivingin Oxygen Deficient waters. In: Internationale Revue Der Gesamten Hidrobiologie. Vol. 67. No. 6: 869 - 886. M anaos (Brasil)

CANTELMO O O. A. \& SOUZA, J.A. 1987. Influência da alimentacão em diferentes níveis protéicos para o desenvolvimento inicial do pacú 
Colossoma mitrei. In: Síntese de trabalhos realizados com espécies do gênero Colossoma. Projeto Aquicultura. Pirassununga S.P.: CPTA

CASTAGNOLLI, N. 1992. Piscicultura de água doce. Faculdade de Ciências A grárias e V eterinárias U NESP. Campus de J aboticabal. SP: U NESP. 189 pp.

DA SILVA, A.B.; DOS SANTOS, E.P.; DE MELO, J.T.C.; SOBRINHO, A.C. \& MELO, F.R. 1984. Quantitative analyses of a preliminary pisciculture experiment of tambaqui, Colossoma macropomum. In: Ciênc. Cult. 36: 82-86. Sao Carlos (Brasil).

ECKMANN, R. 1987. Growth and body composition of juvenile Colossoma macropomum Cuvier 1818 (Characoidei) feeding on artificial diets. In: A quaculture 64: 293 - 303. Instituto de Limnologia, U niv. De Kaustauer. Rep. Federal de Alemania.

FAO, 1985. Relatório de tecnologia e Controle de Qualidade de produtos de pesca. Praia, Rep. de Cabo Verde, 27/11 a 11/12 de 1984. Roma. 24 pp.

GOULDING, M. \& CARVALHO, M.L. 1982. Life history and management of the tambaqui (Colossoma macropomum, Characidae): an important amazonian food fish. In: Rev. bras. zool. 1(2): 107-133. University of California, Press. Berkeley, Los A ngeles, London.

JUNK, W.J. \& HONDA, E.M.S. 1976. A pesca na Amazônia. Aspectos ecológicos e econômicos. Anais do 1 을 Encontro Nacional sobre L imnologia, Piscicultura e Pesca Continental. Belo Horizonte: Fundação João Pinheiro. pp. 211-226.

LESSI, E.; XIMENES-CARNEIRO, A.R. \& LUPÍN, H.M. 1992. Obtenção de ensilado biológico de pescado. In: 2a Consulta de Expertos sobre 
Tecnología de Productos Pesqueros en América Latina. Montevideo, (Uruguay), 11-15 de Diciembre de 1989. Informe de Pesca 441. Supl. Roma. FA 0. 368 pp.

LOVSHIN, L.L. 1980. Situación del cultivo de Colossoma sp. en Sudamérica. En: Rev. Lat. Acuic 5: 27-32. Centro de Pesquisas Ictiologicas Fortaleza. Ceará (Brasil).

MACEDO, E.M. 1979. Necessidades protéicas da nutrição de tambaqui Colossoma macropomum, Cuvier 1818 (Pisces, Characidae). SP: FUCAV, UNESP/J aboticabal. Tese de M estrado, 71 pp.

MCBRIDE，J.R.; IDLER, D.R. \& MACLEOD, R.A. 1968. The liquefaction of British Columbia hering by ensilage, proteolytic enzymes and acids hydrolysis. J. Res. Ed. Can., Ottawa Can, 18(1): 93-112.

M ORI-PINEDO, L.A. 1993. Estudo da possibilidade de substituição do fubá de milho Zea mays L. pela farinha de pupunha Bactris gasipaes H.B.K. em rações para alevinos de tambaqui Colossoma macropomum, Cuvier 1818. Dissertação de M estrado. M anaus (Brasil): IN PA-U FAM I 65pp.

SALDAÑA, A.L. \& LÓPEZ, M.M.E. 1988. Formulación y evaluación de dietas para Colossoma macropomum en M éxico. A n. VI Simp. Lat. e V Simp. Bras. de A quic. Florianópolis. SC. 323 - 344 pp.

SAINT-PAUL, U. 1984. Ecological and physiological investigations of Colossoma macropomum, a new species for fish culture in A mazonia. M ems. A soc. L atinoamericana. In: A cuicult, 5(3): 501-518. U niversitat A nsurg, (West Germany).

SUDEPE/AM. 1988. Sistema de controle de desembarque de pescado em nove municípios do Estado do Amazonas. In: ANUARIO ESTATISTICO DA SUDEPE, MANAUS. 94 pp. 
VAL, A.L. 1986. Hemoglobinas de Colossoma macropomum. Aspectos adaptativos. Tese de Doutorado. Ilhas da Marchantaría, Manaus, Am. INPA/FUA. 112p.

VAN WYK, G.N.; FRANCK, F.; POTGIETER, B.J.; WESSEL, J.P.H. \& ATKINSON, A. 1977. Utilization of fish silage. A study of its consumption by porkers. In: Agroanimalia Pretoria, 9: 13-15. Roma: FAO

VILLELA DE ANDRADE, M.F. 1982. Obtenção de ensilado de resíduos de Sardinella brasiliennsis (Steindachner, 1879) e seu emprego nas formulações de rações de mínimo custo para aves. Dissertação de M estrado. Rio de J aneiro. UFRJ : 113 pp.

WERDER, U., \& SAINT-PAUL, U. 1979. Experiências de alimentação com tambaqui, (Colossoma macropomum), pacú (Mylossoma sp.), jaraquí (Semaprochilodus theropanura) e matrinxã (Brycon melanopterus). In: Acta A mazonica 9(3): 27-36. M anaus (Brasil).

WIGNALL, J. \& TATTERSON, I. 1977. Fish Silage. Process Biochemistry. London, Tropical Products Institute. Jan/Fev. n.p.

XIMENES-CARNEIRO, A.R. 1991. Elaboração e uso de ensilado biológico de pescado na alimentação de alevinos de tambaqui, Colossoma macropomum, (Cuvier, 1818). M anaus: Instituto $\mathrm{N}$ acional de Pesquisas da A mazônia/Fundação U niversidade de Amazonas. Tese de Mestrado. 81 $\mathrm{pp}$. 